\title{
An efficient algorithm for the provision of a day-ahead modulation service by a load aggregator
}

\author{
Sébastien Mathieu, Damien Ernst, Quentin Louveaux \\ University of Liège - Montefiore Institute, B-4000 Liège, Belgium
}

\begin{abstract}
This article studies a decision making problem faced by an aggregator willing to offer a load modulation service to a Transmission System Operator. This service is contracted one day ahead and consists in a load modulation option, which can be called once per day. The option specifies the range of a potential modification on the demand of the loads within a certain time interval. The specific case where the loads can be modeled by a generic tank model is considered.

Under this assumption, the problem of maximizing the range of the load modulation service can be formulated as a mixed integer linear programming problem. A novel heuristic-method is proposed to solve this problem in a computationally efficient manner. This method is tested on a set of problems. The results show that this approach can be orders of magnitude faster than CPLEX without significantly degrading the solution accuracy.

Index Terms-Linear programming, Load management, Load modeling, Optimization methods, Power systems
\end{abstract}

\section{NOMENCLATURE}

The symbols used in this paper are defined below. Others will be defined when used in the text.

\section{A. Parameters}

$M \quad$ Number of controllable loads.

$T \quad$ Number of periods in a market day.

$N \quad$ Duration of the modulation service interval.

$l_{i, t}^{\min } \quad$ Minimal level of tank $i$ at period $t$.

$l_{i, t}^{\max } \quad$ Maximal level of tank $i$ at period $t$.

$\phi_{i, t} \quad$ Outflow of tank $i$ at period $t$.

$p_{i, t}^{\text {min }} \quad$ Minimal power of load $i$ at period $t$.

$p_{i, t}^{\max } \quad$ Maximal power of load $i$ at period $t$.

$\delta_{i} \quad$ Time delay between the supply of electrical power to tank $i$ and its inflow effect.

$a_{i, t}, b_{i, t} \quad$ Linearization coefficients linking inflow and electrical power for tank $i$ at period $t$.

\section{B. Variables}

$o n_{i, t}^{(j)} \quad$ Binary variable equal to 1 if load $i$ is active at period $t$ in scenario $j$.

$l_{i, t}^{(j)}$

$p_{i, t}^{(j)}$ Level of tank $i$ at period $t$ in scenario $j$. Electrical power of tank $i$ at period $t$ in scenario $j$.

$\Delta P_{\max }^{+} \quad$ Maximum amplitude of upward modulation. $\Delta P_{\max }^{-} \quad$ Maximum amplitude of downward modulation.

$\Delta P_{t}^{(j)} \quad$ Variable modulation quantity in scenario $j$ at period $t=j+1, \ldots, j+N$.

\section{INTRODUCTION}

There is an ongoing trend for Transmission System Operators (TSOs) to rely on load modulation services to ensure the safe operation of their system. This trend is caused by two factors: the growth of intermittent renewable generation sources which offer less flexibility in terms of power modulation than dispatchable thermal generation plant [1] and the development of cost-effective solutions for the provision of ancillary services from the demand side [2], [3]. In the liberalized electricity market, the prospect of capitalizing on the value of demand-side flexibility has resulted in the emergence of aggregators. The function of an aggregator is to trade the flexibility in the demand of a large group of electricity consumers via energy and reserve markets [4].

In parallel to the formulation of several variants of the business model of an aggregator, the issue of delivering demand-side flexibility in the electricity market is being addressed in the literature [5]-[9]. Álvarez et al. [5] introduced a methodology for the organization of the flexibility in the demand of a university into services to be traded in the market. The extension of this proposal to commercial consumers and a detailed validation method have also been developed [6]. Ruiz et al. considered thermostatically controlled loads and formulated a control algorithm to achieve a maximum load reduction within a pre-specied time interval [7]. From the perspective of a non-profit coordinating actor, the problem of optimally scheduling the air conditioning demand of the members of a consumer coalition according to the electricity price was addressed in [10]. The optimal day-ahead scheduling of several domestic electrical loads, in light of the uncertainty in the development of dynamic pricing events, as well as in the demand for electricity, was considered in [11].

In the present paper, we focus on the interests of an aggregator providing a 'tailored' load modulation option service to the TSO. The option relates to a maximum load modulation quantity which can be called once a day. More specifically, when the TSO calls its option, the aggregator decreases or increases, according to the TSO's instruction, the total demand of its portfolio of loads over the relevant time interval.

The paper is structured as follows. In Section II, the problem addressed in this paper is carefully stated. Section III describes the load modeling. Section IV recasts the decision problems faced by the aggregator as a MILP model. Section V presents our algorithmic solution to this problem. This algorithm is tested in Section VI. Finally, Section VII concludes. 


\section{Problem statement}

We take the point of view of an aggregator willing to sell to the TSO an option for load modulation. The option, traded on a day-ahead basis, specifies the range of upward $\left(\Delta P^{+}\right)$and/or downward $\left(\Delta P^{-}\right)$load modulation that can be delivered throughout a set $(N \subseteq T)$ of consecutive periods. The service can be deployed by the TSO once per market day, upon notification one period prior to the respective delivery interval. In conformity to the commonly adopted practice for the optional short-term reserve procurement [12], we also assume that the TSO places no constraint on the demand of the controllable loads outside a service delivery interval.

The measurement of the modulation delivery is assumed to happen with respect to the demand of the controllable loads at the point of notification. Therefore, in order to bring a modulation upwards of $\Delta P^{+}$at period $t$, the aggregator measures the total consumption of its load portfolio at period $t-1$ and targets for the next $N$ market periods the same total power with an increment of $\Delta P^{+}$. The adoption of alternative measurement conventions, along the principles of the detailed discussion presented in [13], is straightforward.

This paper address the decision making problem where the aggregator is seeking a load management strategy to maximize the upward and/or downward load modulation range it could sell to the TSO.

\section{LOAD MODELLING}

An aggregator may have different types of loads in its portfolio. We assume that these loads can be accurately represented by a generic tank model, whose inflow is a linear function of the power consumed by the load. The proposed model is similar to storage commitment models used in [8], [9], [14]. Such generic tank model can be potentially applied to several electrical loads such as heaters, heat pumps, fridges, electric cars and pump-tank systems. Let $l_{i, t}$ be the level of the tank during market period $t$. Its level at the beginning of the next market period $l_{i, t+1}$ is given by the following expression:

$$
l_{i, t+1}=l_{i, t}-\phi_{i, t}+\left(a_{i, t-\delta_{i}} p_{i, t-\delta_{i}}+b_{i, t-\delta_{i}}\right) o n_{i, t-\delta_{i}}
$$

The status binary variable $o n_{i, t-\delta_{i}}$ constraints the inflow in the tank to be equal to zero when the load is off. Two main advantages of the generic tank model can be highlighted. First, it can be used to model several flexible loads with energy storage capability. For instance, a heater-room system [15] can be viewed as a tank of temperature, wherein the outflow $\phi_{i, t}$ corresponds to the loss of temperature at period $t$ due to the exchange of thermal energy with the external environment. Coefficients $a_{i, t}$ and $b_{i, t}$ take into account the effect of using the heater on the temperature. The value of $\delta_{i}$ represents the delay that exists between the moment the heater is switched on and the time the temperature starts to increase in the room. Finally, the relevant thermostat lower and upper set-points are given by $l_{i, t}^{\min }$ and $l_{i, t}^{\max }$. The second advantage relates to the linearity of the model. A linear model reduces the complexity of the optimization problem because linear constraints can be handled efficiently. This is particularly beneficial to the problem under consideration since an aggregator may have a large portfolio of loads, which would result in a large number of decision variables.

\section{Mathematical Formulation}

In this section, the problem stated in Section II is mathematically formulated. A critical unknown in the problem is the period in which the TSO calls for the modulation service. The case where the TSO calls for a modulation in period $j \in[1, T]$ is treated as a distinct scenario $j$. Duplicating the number of such scenarios to account for upward and downward modulations and considering the possibility that the TSO may not call for the provision of a modulation as an additional scenario $(j=0)$ results in a set of $2(T-N)+1$ scenarios.

In order to simplify the presentation of the model, we will henceforth refer to the case wherein the TSO only asks for modulation upwards by restricting the mathematical formulation to a subset of the problem scenarios. The extension of the model to both types of modulation is straightforward.

The maximization of the upward modulation quantity can be expressed as the following optimization problem :

$$
\max \Delta P_{\max }^{+}
$$

subject to :

for $j=1, \ldots, T-N, t \in\{j+1, \ldots, j+N\}$,

$$
\begin{gathered}
\sum_{i=1}^{M} p_{i, t}^{(j)}=\sum_{i=1}^{M} p_{i, j}^{(j)}+\Delta P_{t}^{(j)} \\
\Delta P_{\max }^{+}<\Delta P_{t}^{(j)}
\end{gathered}
$$

for $i=1, \ldots, M, j=0, \ldots, T-N, t=\delta_{i}, \ldots, T+1+\delta_{i}$,

$$
l_{i, t+1}^{(j)}=l_{i, t}^{(j)}-\phi_{i, t}+a_{i, t-\delta_{i}} p_{i, t-\delta_{i}}^{(j)}+b_{i, t-\delta_{i}} o n_{i, t-\delta_{i}}^{(j)}
$$

for $i=1, \ldots, M, j=0, \ldots, T-N, t=1, \ldots, \delta_{i}-1$,

$$
l_{i, t+1}^{(j)}=l_{i, t}^{(j)}-\phi_{i, t}
$$

for $i=1, \ldots, M, t=1, \ldots, T+1+\delta_{i}, j=0, \ldots, T-N$,

$$
\begin{aligned}
\min _{i, t} & \leq l_{i, t}^{(j)} \leq \max _{i, t} \\
p_{i, t}^{\min } \text { on }_{i, t}^{(j)} & \leq p_{i, t}^{(j)} \leq p_{i, t}^{\max } \text { on }_{i, t}^{(j)}
\end{aligned}
$$

for $i=1, \ldots, M, t=1, \ldots, T+1+\delta_{i}, j=1, \ldots, T-N$ such as $t \leq j$,

$$
\begin{gathered}
l_{i, t}^{(j)}=l_{i, t}^{(0)} \\
p_{i, t}^{(j)}=p_{i, t}^{(0)} \\
o n_{i, t}^{(j)}=o n_{i, t}^{(0)}
\end{gathered}
$$

and $l_{i, t}^{(j)}, p_{i, t}^{(j)}, \Delta P_{t}^{(j)}, \Delta P_{\max }^{+}$continuous, $o n_{i, t}^{(j)}$ binary.

Problem (2)-(11) is a typical MILP problem. Constraint (3) links the individual power consumed by each load with a modulation upwards with respect to the total power consumed at period $j$. Inequality (4) states that the maximum modulation quantity that can be achieved during $N$ periods is the minimum of the quantity at each period. Equalities (5) and (6) define the 
tank level dynamics. Constraint (7) imposes the bounds of the levels of the fictive tanks, while (8) expresses bounds on the power consumed by each load. Equalities (9), (10) and (11) express non-anticipativity constraints. Considering a period $t$ and two scenarios $j_{1}>t$ and $j_{2}>t$, this implies that in these two scenarios, no modulation has occurred by period $t$. Hence all variables for these scenarios in period $t$ must be equal.

Finally, the model related to the maximization of the range of load modulation, has the following objective function:

$$
\max \left(\Delta P_{\max }^{+}+\Delta P_{\max }^{-}\right)
$$

The additional constraints referring to the downwards modulation service are directly obtained from those presented previously by considering the complete set of scenarios.

\section{HEURISTIC ALGORITHM}

Branch and bound [16] based software are the most established tools for solving MILP problems. However, the use of such software becomes computationally too demanding for large-sized problems as it may be the case here since an aggregator may have hundreds, if not thousands, of loads in its portfolio. This heuristic algorithm of polynomial complexity finds a good solution in a small amount time. The proposed algorithm is split in the following three steps :

$A$. Check feasibility to ensure than the problem leads to at least one solution.

$B$. Build an initial solution which ensures that each load's level and power are within their bounds at every period.

$C$. Improve the solution to increase the objective value by iterated local search [17], [18].

\section{A. Check feasibility}

The first algorithmic step is to check whether the problem parameters leads to a feasible solution. The sequential nature of the load model allows us to propagate the bounds on the level from one period to the following ones. For each load $i$, at least one activation scheme must be feasible such that the level of the tank can be between $l_{i, t}^{\min }$ and $l_{i, t}^{\max }$ for every period $t$. The complete set of conditions that needs to be satisfied in order to have a feasible problem are given by (13)-(16).

$$
l_{i, t}^{\max } \geq l_{i, t-1}^{\min }-\phi_{i, t-1}+a_{i, \tau} l_{i, \tau}+b_{i, \tau}
$$

or

$$
\begin{gathered}
l_{i, t}^{\min } \leq l_{i, t-1}^{\max }-\phi_{i, t-1} . \\
l_{i, t}^{\max } \geq l_{i, k}^{\min }-\sum_{\tau=k}^{t-1} \phi_{i, \tau} \forall k \in[1, t-1] . \\
l_{i, t}^{\min } \leq l_{i, k}^{\max }+\sum_{\tau=k}^{t-1}\left(a_{i, \tau-\delta_{i}} p_{i, \tau-\delta_{i}}^{\max }+b_{i, \tau-\delta_{i}}-\phi_{i, \tau}\right)
\end{gathered}
$$

$\forall k \in[1, t-1]$ with $\tau=t-1-\delta_{i}$.

\section{B. Build an initial solution}

The next step of the algorithm is to determine an activation scheme for each load, i.e. to define a feasible power demand profile for each load without considering the provision of upward/downward modulations. Such profile is built on an ascending period order. For every period $t$, the algorithm starts by arbitrarily selecting an approximation of the total power consumed by the loads $\Theta_{t}$. This value is chosen as a fraction of the maximal power that can be consumed by the $M$ loads. Once $\Theta_{t}$ is defined, the algorithm assigns power to every load by trying to stay as close as possible to the target value $\Theta_{t}$. The full procedure is described in Algorithm 1.

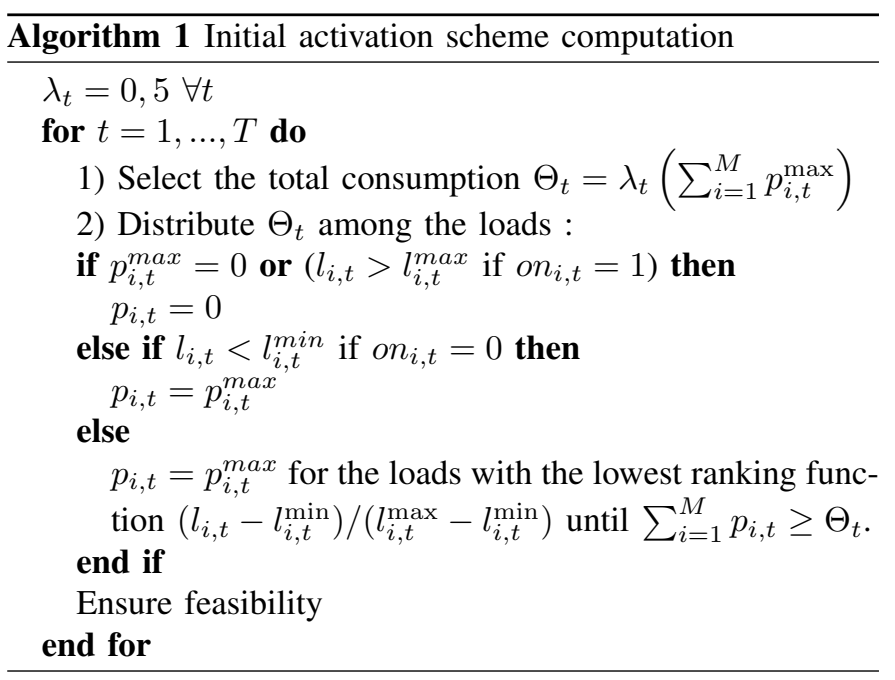

This procedure does not ensure the feasibility of the solution. Because $l_{i, t}^{\min }$ and $l_{i, t}^{\max }$ are not constant over all periods, the level of each load is not necessarily within the level bounds and therefore, the solution built may not be feasible. To recover feasibility at period $t$ for load $i$, the heuristic use a feasibility recovery algorithm. For the sake of conciseness, we explain the case where the level violates a lower bound. The case of exceeding the upper bound can be treated similarly. A level violation is defined as $\Delta l_{i}=l_{i, t}^{\min }-l_{i, t}$ which is positive if the level at period $t$ is lower than the minimum level for this period. The power consumed by the load is changed in previous periods to get a feasible level of load $i$ at period $t$. The details of the algorithm are explained in the Algorithm 2.

So far, the heuristic has built a feasible solution that does not take into account the possibility of a call for modulation by the TSO. The next step is to build an activation scheme for the scenarios under which modulation is provided. For each scenario $j \geq 1$, the production pattern must remain the same for periods $t \leq j$ as the initial solution. For the $N$ modulation delivery periods, the modulation type dictates the decisions to be made. For a modulation upwards, every load is activated at its maximal power. For the modulation downwards, every load is switched off. This assignment may lead to some infeasibilities with respect to the level constraints. They are corrected by using the Algorithm 2. 


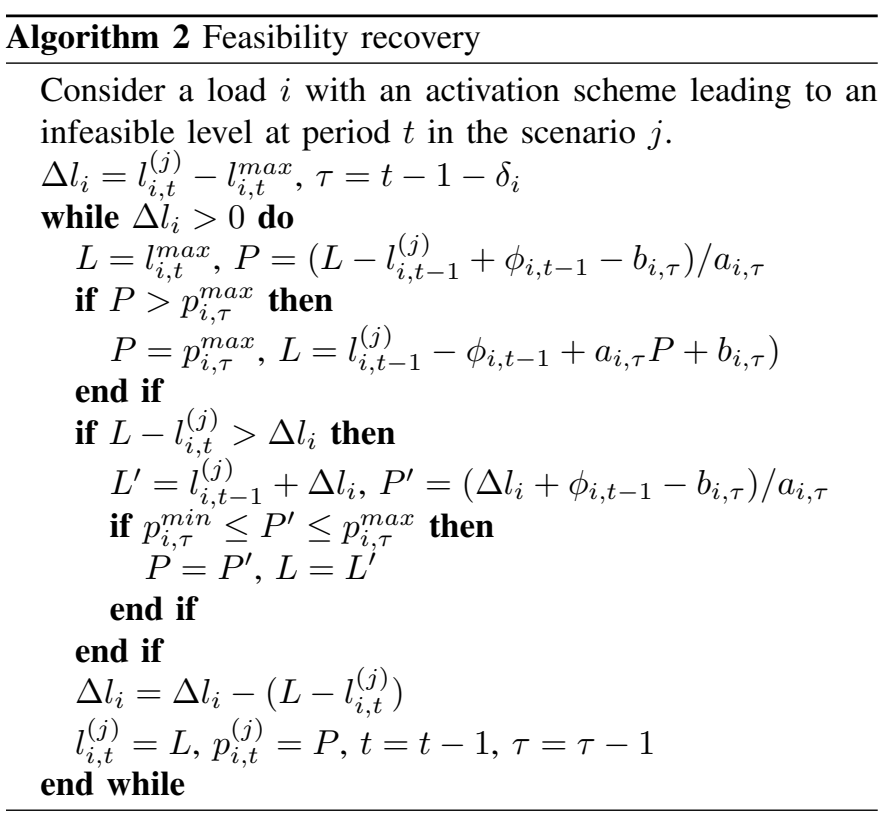

\section{Improve the solution}

This section focuses on the possibility to improve drastically the initial solution thanks to iterated local search [17], [18]. Iterated local search tries to improve a feasible solution by applying a perturbation to find a new solution in the neighbourhood of the feasible solution. Improvement is done by varying the value $\lambda_{t} \in[0,1]$ used in Algorithm 1 to quantify the share of active consumption at period $t$ with respect to the. The complete procedure is described in the Algorithm 3.

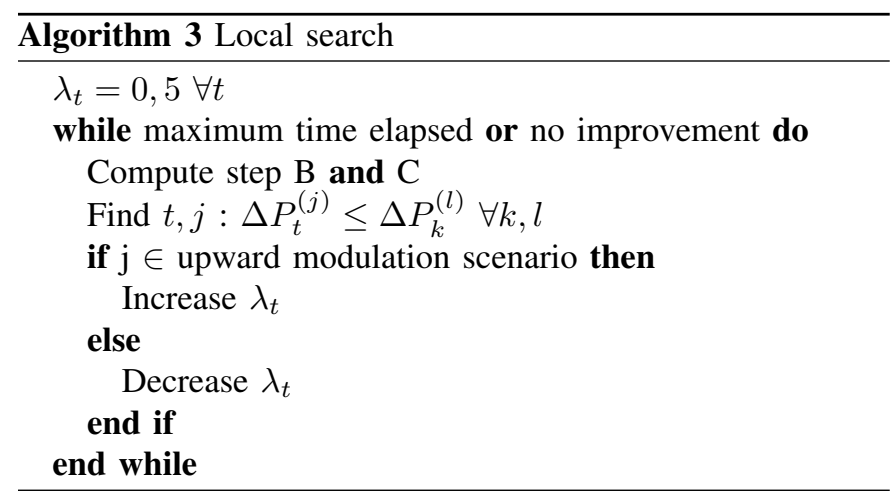

The overall algorithm complexity is $M T \log (M)+M T^{3}$ in the worst case and can fall down to $M T \log (M)+M T^{2}$ for each iteration of the local search.

\section{Simulation Results}

This section gathers and discusses our simulation results. It starts with a description of the mechanism used for generating the test problems in Section VI-A. A comparison of the proposed algorithm with CPLEX is presented in Section VI-B.

\section{A. Mechanism for generating the test problems}

For every test problem, an option service lasts $N=1$ period and the aggregator wants to maximize the sum of downwards and upwards modulation quantities it can sell. A market day starts at 00:00 am and finishes at the same hour the next day.

A portfolio includes $25 \%$ of electric heaters and $75 \%$ of electric vehicles. The mechanism used for generating the loads for every test problem with $M$ loads amounts to calling $0.75 \times M$ times the procedure build_ev and $0.25 \times M$ times the procedure build_heater. These procedures are given below. build_heater creates a generic tank model that corresponds to a heater with the parameters of Table I. build_ev creates a generic tank model corresponding to an electric car with the parameters of Table I. A number is drawn for every market period from a Bernouilli distribution with $p=0.95$. If this number is equal to 1 , the vehicle is plugged else it is unplugged. To determine the outflow of the vehicle when it is unplugged, a number in the interval $[0,90 \%]$ is drawn at random.

TABLE I

\begin{tabular}{c|cc|cc}
\hline $\begin{array}{c}\text { Load Type } \\
\text { State }\end{array}$ & Electric Heater & \multicolumn{2}{|c}{ Electric Vehicle } \\
a.m.- 4.30 p.m. & Else & On-plug & Unplugged \\
\hline$l_{i, t}^{\text {min }}$ & $17^{\circ}$ & $5^{\circ}$ & \multicolumn{2}{|c}{$10 \%$} \\
$l_{i, t}^{\text {max }}$ & $23^{\circ}$ & $17^{\circ}$ & \multicolumn{2}{|c}{$100 \%$} \\
$\phi_{i, t}$ & $1^{\circ} / h$ & & $0 \%$ & {$[0,90 \%]$} \\
$p_{i, t}^{\text {min }}$ & $2 k W$ & & $3 k W$ & 0 \\
$p_{i, t}^{\text {max }}$ & $8 k W$ & $3 k W$ & 0 \\
$\delta_{i}$ & 1 & \multicolumn{2}{c}{0} \\
$a_{i, t}$ & $2^{\circ} / k W h$ & & $7.5 \% / k W h$ & 0 \\
$b_{i, t}$ & $0^{\circ} / h$ & & $-2 \% / h$ & 0 \\
\hline
\end{tabular}

\section{B. Comparison with CPLEX}

The heuristic is compared with the CPLEX tool version 12.4 on 500 problems with 32 market periods and 100 loads, each being generated according to the mechanism described in $\mathrm{VI-A}$. The relative MIP gap tolerance parameter of CPLEX is set 0.4 to stop it when it finds an acceptable feasible solution.

The algorithms are compared by computing the ratio of the sum of the upward and downward modulation quantities the aggregator can sell. The results are reported in a concise way by the histogram of ratios shown in Fig. 1. As can be seen, the solutions provided by the two algorithms are close. The heuristic algorithm is however much faster than CPLEX as shown by Fig. 2 that reports, using histograms, the computing times of both algorithms on the 500 test problems. Note that these computing times have been obtained by using as processor an Intel Core i7 with a clock rate of $3.33 \mathrm{GHz}$.

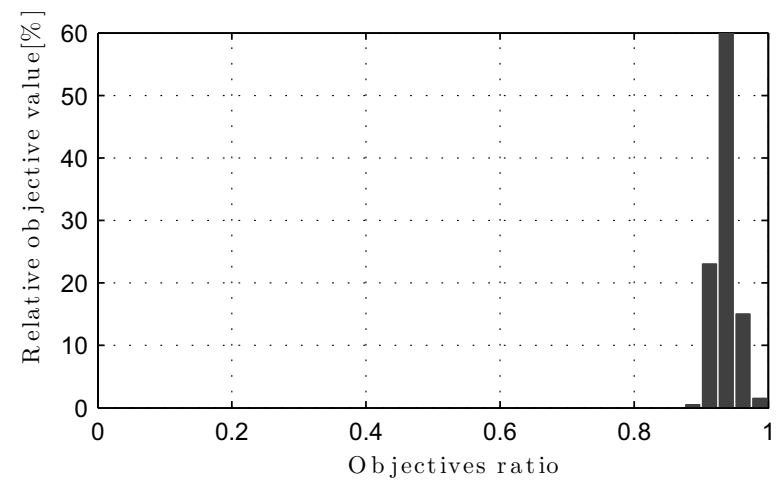

Fig. 1: Histogram of the ratios between the solutions obtained by the heuristic algorithm and CPLEX for 500 test problems. 


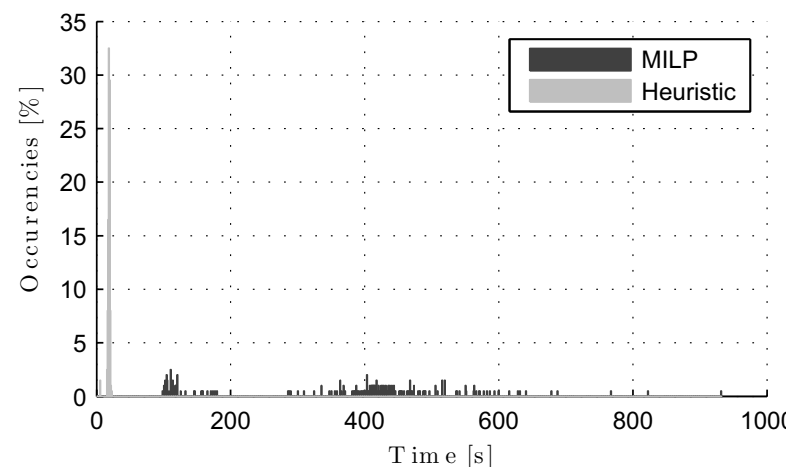

Fig. 2: Histograms of the computing times of our heuristic algorithm and of CPLEX for 500 test problems.

\section{CONCLUSION}

In this paper, we have studied the decision problem faced by an aggregator willing to sell an option for a specific type of load modulation service to a TSO. The service takes the form of an upward and/or downward load modulation capacity, which can be used by the TSO once per day. The problem was formalized as a classical MILP problem. For solving this problem, we have proposed a customized algorithm that has been shown to be much faster than CPLEX, while still leading to high-quality solutions. Further research may adapt the algorithm to alternative load models, i.e. non-linear models. In this respect, it would also be worth considering stochastic load models, even if those considerably complexify the decisionmaking problem faced the aggregator. Another interesting line of research may focus on the case wherein the modulation capacity traded between the TSO and the aggregator can be distinctively defined for every single market period. Note that such formulation will imply considering the payback effect following the delivery of a load modulation.

\section{ACKNOWLEDGMENTS}

This research is supported by the public service of Wallonia Department of Energy and Sustainable Building within the framework of the GREDOR project. The authors thank the financial support of the Belgian Network DYSCO, an Interuniversity Attraction Poles Program initiated by the Belgian State, Science Policy Office. The authors also thank the Powerdale company for fruitful discussions.

\section{REFERENCES}

[1] F. Bouffard and M. Ortega-Vazquez, "The value of operational flexibility in power systems with significant wind power generation," in Power and Energy Society General Meeting, 2011 IEEE, 2011, pp. 1 -5.

[2] D. S. Callaway, "Tapping the energy storage potential in electric loads to deliver load following and regulation, with application to wind energy," Energy Conversion and Management, vol. 50, no. 5, pp. 1389-1400, 2009.

[3] K. Pandiaraj, P. Taylor, N. Jenkins, and C. Robb, "Distributed load control of autonomous renewable energy systems," IEEE Transactions on Energy Conversion, vol. 16, no. 1, pp. 14-19, 2001.
[4] M. Negnevitsky, T. D. Nguyen, and M. de Groot, "Novel business models for demand response exchange," in Power and Energy Society General Meeting, 2010 IEEE, 2010, pp. $1-7$.

[5] C. Álvarez, A. Gabaldón, and A. Molina, "Assessment and simulation of the responsive demand potential in end-user facilities: application to a university customer,' IEEE Transactions on Power Systems, vol. 19, no. 2, pp. 1223-1231, May 2004.

[6] M. Alcázar-Ortega, G. Escrivá-Escrivá, and I. SeguraHeras, "Methodology for validating technical tools to assess customer demand response: application to a commercial customer," Energy Conversion and Management, vol. 52, no. 2, pp. 1507-1511, 2011.

[7] N. Ruiz, I. Cobelo, and J. Oyarzabal, "A direct load control model for virtual power plant management," Power Systems, IEEE Transactions on, vol. 24, no. 2, pp. 959-966, 2009.

[8] J. Taylor, D. Callaway, and K. Poolla, "Inventory control of storage in distribution systems," in American Control Conference (ACC), 2012, IEEE, 2012, pp. 2147-2152.

[9] J. Kim and W. Powell, "Optimal energy commitments with storage and intermittent supply," Operations research, vol. 59, no. 6, pp. 1347-1360, 2011.

[10] D. Menniti, F. Costanzo, N. Scordino, and N. Sorrentino, "Purchase-bidding strategies of an energy coalition with demand-response capabilities," IEEE Transactions on Power Systems, vol. 24, no. 3, pp. 1241-1255, 2009.

[11] M. Pedrasa, E. Spooner, and I. MacGill, "Robust scheduling of residential distributed energy resources using a novel energy service decision-support tool," in Innovative Smart Grid Technologies (ISGT), 2011 IEEE PES, 2011, pp. $1-8$.

[12] S. Matthews and I. Kockar, "New Short-TermOperation-Reserve Services in the UK electricity market," in Power Engineering Society General Meeting, 2007. IEEE, 2007, pp. $1-7$.

[13] M. Goldberg, "Measure twice, cut once," Power and Energy Magazine, IEEE, vol. 8, no. 3, pp. 46-54, 2010.

[14] Y. Xu, L. Xie, and C Singh, "Optimal scheduling and operation of load aggregators with electric energy storage facing price and demand uncertainties,' in North American Power Symposium (NAPS), 2011, IEEE, 2011, pp. 1-7.

[15] V. Bakker, M. Bosman, A. Molderink, J. Hurink, and G. Smit, "Demand side load management using a three step optimization methodology," First IEEE International Conference on Smart Grid Communications, SmartGridComm 2010, 2010.

[16] L. Wolsey, "Integer programming," IIE Transactions, vol. 32, pp. 273-285, 2000.

[17] G. Kochenberger, Handbook of metaheuristics. Springer, 2003.

[18] R. Battiti, M. Brunato, and F. Mascia, Reactive search and intelligent optimization. Springer, 2008, vol. 45. 